\title{
Discovery of Highly Insecticidal Synthetic Spinosyn Mimics - CAMD Enabled De Novo Design Simplifying a Complex Natural Product \\ For: Pest Management Science - Spotlight
}

Thomas C Sparks, Gary D. Crouse ${ }^{\S}$, David Demeter, Geno Samaritoni" ${ }^{\#}$ Casandra L. McLeod ${ }^{\circledR}$, Corteva Agriscience ${ }^{\mathrm{TM}}$, Agriculture Division of DowDuPont ${ }^{\mathrm{TM}}$, Dow AgroSciences. Discovery Research 9330 Zionsville Road, Indianapolis, IN 46268

Phone: 317-337-3064;

${ }^{*}$ Corresponding Author (T.C.S.)

Fax: 317-337-3205,

E-mail: tcsparks@dow.com

${ }^{\S}$ Current address

5069 E 146th St

Noblesville IN 46062

Indianapolis, IN

E-mail: gdcrouse1088@gmail.com

"Current address

Department of Chemistry and Chemical Biology, Indiana University - Purdue University

Indianapolis, 402 N. Blackford Street, Indianapolis, IN 46202

Phone: 317-274-6804

E-mail: jsamarit@iupui.edu

${ }^{\&}$ Current Address

6034 Haverford Ave.

Indianapolis, IN 46220

Phone: $317-775-4454$

E-mail: cmcleod@kippindy.org

Abstract:

Simplifying complex natural products: Computer modeling-based design leads to highly insecticidal, chemically simpler synthetic mimics of the spinosyn natural products that are active in the field.

This is the author's manuscript of the article published in final edited form as:

Sparks, T. C., Crouse, G. D., Demeter, D., Samaritoni, G., \& McLeod, C. L. (2018). Discovery of Highly Insecticidal Synthetic Spinosyn Mimics - CAMD Enabled De Novo Design Simplifying a Complex Natural Product. Pest Management Science, 0(ja). https://doi.org/10.1002/ps.5217 


\section{Introduction}

Natural products (NP) have been and continue to be a source of products, starting materials and inspiration for the discovery and development of new chemical tools for crop protection ${ }^{1-3}$ and pharmaceuticals. ${ }^{4}$ There are numerous examples of NPs serving as inspiration for new agrochemicals, the most significant (market share) NP-based classes today being the synthetic pyrethroids and the strobilurins which currently represent $16 \%$ and $31 \%$ of the insecticide and fungicide markets, respectively. ${ }^{3} \quad$ However, the most important naturally occurring NPs currently in use directly as crop protection tools are the avermectins (abamectin) and spinosyns (spinosad 1), 2,3 both naturally occurring insecticidal macrocyclic lactones. These insecticidal macrolides are large complex molecules, represent novel areas of chemistry and, importantly, unique modes of action. ${ }^{5,6}$ Also, both of these macrolides have been subject to extensive postfermentation modification programs leading, in turn, to semi-synthetic derivatives including emamectin benzoate ${ }^{7,8}$ and spinetoram $4^{9}$ which have themselves been developed as products, ${ }^{7,10}$ possessing improved insecticidal efficacy and expanded spectra. In this way, these insecticidal macrolides show parallels with pharmaceutical antibiotic counterparts such as erythromycin and its semi-synthetic derivative azithromycin. ${ }^{11}$

As already noted, NPs have served as inspiration for a wide range of pesticides. ${ }^{1,2}$ However, unlike other NPs, commercially viable synthetic mimics of the large macrolides have been unknown for agrochemicals and, as far as can be determined, pharmaceuticals. We recently reported that simplified synthetic mimics of the complex spinosyn structure are indeed possible, exhibiting laboratory efficacy near that of spinetoram. ${ }^{12,13}$ Herein we provide further elaboration on the discovery and development of synthetic spinosyn mimics, resulting in highly active, photostable analogs that demonstrate excellent in vivo efficacy in the laboratory and field.

\section{Design of Synthetic Spinosyn Mimics}

The spinosyns, which include spinosad 1 and spinetoram 4 (Fig. 1), are class of large, complex macrolide natural products (NPs) that are highly insecticidal towards a broad spectrum of lepidopteran and other insect pests. Interestingly, they also exhibit very favorable toxicological and environmental profiles, both molecules receiving the EPA Green Chemistry awards. ${ }^{10}$ The structure activity relationships (SAR) of the naturally occurring spinosyns and a wide range of semi-synthetic derivatives have been extensively explored. ${ }^{10,14-16}$ These studies, guided by an 
artificial intelligence-based analysis of the spinosyn quantitative structure activity relationships, ${ }^{9}$ ultimately lead to the discovery of spinetoram 4, a semi-synthetic derivative of spinosad 1 that retained the macrolide-based tetracycle core. However, in spite of the discovery and development of spinetoram 4, and the synthesis of more than 1000 analogs, further commercial exploitation of this chemistry was limited due the inherent complexity of the spinosyn macrolide tetracycle. Hence, the idea of synthetic spinosyn mimics was considered.

The synthesis of the spinosyn tetracycle has been demonstrated, but the syntheses are long and complex. ${ }^{17,18}$ Likewise, there have been some efforts to simplify parts of the tetracycle, but the fundamental structure of a large tetracycle remains. ${ }^{19}$ An early notion for a synthetic spinosyn envisioned a simple, rigid skeleton that could provide points of attachment for the two spinosyn sugar moieties, placing them in the correct geometry in 3D space; a concept later tested, but less effective than the path ultimately taken. Another simpler approach was to use computer-aided molecular design (CAMD) to essentially reverse engineer the spinosyn structure by seeking potential scaffolds that could potentially fill the space of the tetracycle and allow for the addition of a linker to attach the rhamnose sugar, which was viewed as key for biological activity. Provision was also made for potentially emulating all or part of the forosamine sugar. The hope was that the resultant molecule would be simpler than the complex molecular structure of spinosyns, open to further modification and optimization, and perhaps have different, exploitable physical properties. Initial CAMD modeling of potential cores suggested that the pyridobenzimidazole (PBI) core structure (Fig. 1) might be a suitable starting point ${ }^{13}$ as it overlaid well with the spinosyn tetracycle. Further CAMD-based modifications to the PBI-core included adding a 2',3',4'-tri-O-methyl rhamnose sugar via an indane linker to correctly place the rhamnose in its putative binding pocket and overlay with the rhamnose moiety of the spinosyns and a $\mathrm{N}, \mathrm{N}$-dimethyl amine to mirror the forosamine functionality. Although the resulting molecule 5 (Fig. 1) appeared to overlay well with spinosyn A (Fig. 2), it proved synthetically very challenging and when finally completed it was found to be virtually inactive. Conversely, the synthetic precursor (6) to the original target (5) proved to be of greater interest. The precursor molecule 6 (Fig. 1) also overlaid nicely with spinosyn A, placing the rhamnose sugar in the correct position (Fig. 2). ${ }^{13}$ Disappointingly, when 6 was evaluated using a standard in vivo diet bioassay, it was inactive. Because an in vitro binding assay for the spinosyns was not available, it was not possible to attribute the lack of activity to poor interaction at the spinosyn binding site, lack of structural components (i.e. forosamine), or to some other limitation such as poor uptake or metabolism. To better understand if the lack of activity was inherent in 
the molecule, an injection assay was used as a substitute for an in vitro binding assay. Surprisingly, when 6 was injected into larvae of Spodoptera exigua (beet armyworm), within minutes the treated larvae began to exhibit symptoms consistent with intoxication by spinosyns. These symptoms included tremors, paralysis and rapid movement of mouth parts. ${ }^{13}$ Interestingly, the modeling suggested two potential ways to overlay analog 6 with the structure of spinosyn $A$; the first put the structure of 6 into the space occupied by the forosamine (not shown) similar to that proposed for analog 5 (Fig. 2), while the second put 6 into the space around the C21 position (Fig. 2). As noted above, when tested in the injection assays, analog 5 showed little in the way of symptoms, suggesting that the addition of an $N, N$-dimethyl amine only served to greatly decrease activity relative to analog 6 . These observations highlighted the idea that the alignment of analog 6 through the $\mathrm{C} 21$-position was likely the more relevant motif.

The observation of spinosyn-like symptoms was enough to fuel further exploration around this initial structure using further CAMD-based investigations and bioactivity directed synthesis, initially all driven by symptomology. ${ }^{13}$ These efforts led to a series of molecules that ultimately resulted in analog 7 (Fig.1) which retained the indane-rhamnose configuration of analog 6, but simplified the $\mathrm{PBI}$ core with an aryl-pyrimidine moiety. Analog 7 represented the first synthetic spinosyn motif to exhibit in vivo insecticidal activity (Table 1). The structural evolution leading to analog 7 was a lengthy process requiring thousands of larval injections over a period of two years. The in vivo insect activity of analog 7 led to further optimization around the tri-aryl nucleus, including replacement of the indane linker with an oxime and replacing the pyrimidine with alternative heterocycles, leading ultimately to a triazole. Finally, extensive analog synthesis investigating a range of substituents around the two phenyl rings of the tri-aryl ring system led to the incorporation of a $p-\mathrm{CF}_{3} \mathrm{CF}_{2} \mathrm{O}$ - substituent on the aryl A-ring (Ar-A), leading to analog 8 (Fig. 1). Analog 8 also incorporated lessons from the spinosyn $S A R^{14,15}$ by incorporating an O-ethyl in the 3'-position of the rhamnose (Fig. 1), providing a further boost in insecticidal potency. ${ }^{12}$ Analog $\mathbf{8}$ displayed excellent in vivo activity against lepidopteran insect pests such as $S$. exigua and Helicoverpa zea (corn earworm) that was equal to spinetoram (Table 1). However, greenhouse tests showed that analog 8 with the oxime linker was not photostable (Table 1), showing a substantial decline in efficacy after a few days of UV exposure. This UV greenhouse data stimulated further structural exploration resulting in replacement of the oxime-linker of analog $\mathbf{8}$ with a carbamate-linker to yield analog $\mathbf{9}$, which retained insecticidal efficacy similar to spinetoram for the lepidopteran species tested (Table 1). Importantly, analog 9 also maintained its insecticidal activity even under UV conditions (Table 1). These observations were further 
validated in field tests where analog 9 was found to be as effective as spinetoram against larvae of Trichopluisa ni (cabbage looper) (Fig. 3).

\section{Mode of Action}

In light of the very significant structural departure of the synthetic spinosyn mimics from that of the spinosyns, the obvious question of the mode of action arises. The molecular target site of the spinosyns is an allosteric site associated with the a6 subunit of the insect nicotinic acetylcholine receptor ( $\mathrm{nAChR}$ ), with no cross-resistance to other modes of action. ${ }^{16,20}$ As already noted, an in vitro target site binding assay for the spinosyns was not available. However, resistance to the spinosyns is primarily due to alterations or point mutations in the $\alpha 6$ subunit of the nAChR. ${ }^{16}$ A strain of Drosophila melanogaster (fruit fly) highly resistant to the spinosyns and possessing an altered $\mathrm{D} \alpha 6 \mathrm{nAChR}{ }^{20}$ was used to explore the mode of action of the spinosyn mimics. Analogs $\mathbf{8}$ and $\mathbf{9}$ were both found to be highly cross-resistant in this spinosyn resistant strain (Table 1 ), both at levels similar to spinosad and spinetoram, indicating the strong likelihood that $\mathbf{8}$ and $\mathbf{9}$ possess the same mode of action as the spinosyns.

\section{Summary}

Our studies show that the large, complex macrolide tetracycle of the spinosyns can be effectively emulated by a simple tri-aryl framework. Importantly, these new synthetic spinosyn mimics appear to possess the same mode of action of the spinosyns, can be more active than the NP (spinosad), and are as active in the field as the more recent semi-synthetic spinosyn derivative, spinetoram. An unexpected observation with these new synthetic spinosyn mimics is that based on the overlays with spinosyn A (Fig. 2), the forosamine sugar appears to be outside the synthetic mimic motif, suggesting that the forosamine sugar or its equivalent bioisostere is not essential for insecticidal activity for these novel molecules. This observation is in marked contrast to the spinosyn NPs where loss of the forosamine leads to a dramatic loss in insecticidal activity. ${ }^{10,15,16}$ Interestingly, the binding space around the C21-position of the spinosyns appears tolerant of fairly large substituents as evidenced by the good insecticidal activity of spinosyn analogs possessing a range of C21 substitutions far larger than the ethyl group of spinosad including butenyl, styrene, cyclobutyl, etc. ${ }^{21,22}$ Thus, the likely alignment of the synthetic spinosyns mimics into this space is consistent with what is known regarding the spinosyn SAR. Importantly, and as noted previously, these new synthetic spinosyn mimics represent the first time synthetic mimics with efficacy equal to or better than their natural counterparts have been discovered for any agricultural macrolide NP and, as far as can be 
ascertained, in the pharmaceutical arena as well. Implicit in our results is the idea that it may also be possible to simplify other macrolide-based compounds including macrolide antibiotics and other crop protection products, presenting new options for exploiting novel NP modes of action with attributes outside of those observed for the NP, including different physical properties, enhanced efficacy, and perhaps altered spectrum. Although the resulting synthetic spinosyn mimics did not quite achieve the reduction in molecular size envisioned, they are still smaller and chemically simpler than the spinosyn NPs. An issue with the prior spinosyn mimic (8) was the UV-instability of the oxime linker - the carbamate bioisostere solves that problem leading to a synthetic spinosyn mimic (9) that is both more photostable and active in the field. Thus, the idea of devising chemically simpler mimics of the large spinosyn macrolides has gone from concept to reality.

Acknowledgements. We thank Cathy Young, Melissa Harris and Ricky Hunter for bioassay support, Dr. Melissa Siebert for the field test data, and Mr. Jerry Watson and Drs. Debra Camper, Ronda Hamm, James Hunter, and Michael Loso for very useful comments and discussions. We also thank the editor and the anonymous reviewers for their very helpful comments and suggestions.

\section{References}

1. Cantrell CL, Dayan FE and Duke SO, Natural products as source for new pesticides, J. Nat Prod. 75:1231-1242 (2012).

2. Gerwick BC and Sparks TC, Natural products for pest control: an analysis of their role, value and future, Pest Manag. Sci., 70, 1169-1185 (2014).

3. Sparks TC, Hahn DR, Garizi NV, Natural products, their derivatives, mimics and synthetic equivalents: Role in agrochemical discovery, Pest Manag. Sci., 73, 1169-1185 (2017).

4. Newman DJ, Cragg GM, Natural products as sources of new drugs from 1981 to 2014, J Nat. Prod., 79, 629-661 (2016).

5. Sparks TC and Nauen R, IRAC: Mode of action classification and insecticide resistance management, Pestic. Biochem. Physiol. 121, 122-128 (2015).

6. Yu S, The Toxicology and Biochemistry of Insecticides, $2^{\text {nd }}$ ed., CRC Press, Boca Raton, FL. (2015).

7. Kornis Gl, Avermectins and milbemycins, In Agrochemicals from Natural Products, ed. by Godfrey, CRA, Marcel Dekker, New York, pp. 215-255 (1995). 
8. Pitterna T, Chloride channel activators / new natural products: Avermectins and milbemycins, In Modern Crop Protection Compounds Vol. 3: Insecticides, $2^{\text {nd }}$ ed, ed. by Krämer W, Schirmer U, Jeschke P, Witschel M, Wiley-VCH, Weinheim, GR, pp. 1305-1326 (2012).

9. Sparks TC, Crouse GD, Dripps JE, Anzeveno P, Martynow J, DeAmicis CV, Gifford J, Neural network-based QSAR and insecticide discovery: spinetoram, J. Comput. Aided Mol. Des. 22:393-401 (2008).

10. Dripps JE, Boucher RE, Chloridis A, Cleveland CB, DeAmicis CV, Gomez LE, Paroonagian DL, Pavan LA, Sparks TC, Watson GB, The spinosyn insecticides, In Green Trends in Insect Control_ed. by Lopez O, Fernandez-Bolanos JG, RSC Publishing, Cambridge, UK, pp. 163-212 (2011)

11. Jelic $\mathrm{D}$, Antolovic $\mathrm{R}$, From erythromycin to azithromycin and new potential ribosome-binding antimicrobials, Antibiotics, 5, 29 (2016).

12. Sparks TC, Crouse GD, Demeter DA, Brown A, Bryan K, Samaritoni JG, Insecticide discovery - Synthetic spinosyn mimics, Abstract, Agro 388, 254 ${ }^{\text {th }}$ American Chemical Society National Meeting, Washington DC, Aug. 20-24 (2017).

13. Crouse GD, Demeter DA, Samaritoni G, McLeod, CL, Sparks TC, De novo design of potent insecticidal synthetic mimics of spinosyn macrolide natural products, Sci. Reports, 8:4861 (2018).

14. Crouse GD, Sparks TC, Schoonover J, Gifford JM, Bruce T, Worden TV, Martynow JG, Recent advances in the chemistry of the spinosyns. Pest Management Sci. 57, 177-185 (2001).

15. Sparks TC, Crouse GD, Durst G, Natural products as insecticides: The biology, biochemistry and quantitative structure activity relationships of spinosyns and spinosoids. Pest Management Sci. 57, 896-905 (2001).

16. Geng C, Watson GB, Sparks TC, Nicotinic acetylcholine receptors as spinosyns targets for insect pest management, In Target Receptors in the Control of Insect Pests: Part I, Advances in Insect Physiology, Vol. 44, E. Cohen, ed., Academic Press, pp. 101-210 (2013).

17. Paquette LA, Collado I, Purdie M, Total synthesis of spinosyn A. 2. Degradation studies involving the pure factor and its complete reconstitution, J. Am. Chem. Soc. 129, 25532562 (1998).

18. Mergott DJ, Frank SA, Roush WR, Total synthesis of (-)-spinosyn A, PNAS 101, 1195511959 (2004). 
19. Tietze LF, Brasche G, Grube A, Bohnke N, Stadler C, Synthesis of novel spinosyn A analogues by Pd-mediated transformations, Chem Eur. J. 13, 8543-8563 (2007).

20. Watson GB, Chouinard SW, Cook KR, Geng C, Gifford JM, Gustafson GD, Hasler JM, Larrinua IM, Letherer TJ, Mitchell JC,Pak WL, Salgado VL, Sparks TC, Stilwell GE, Heterologus expression of a spinosyn-sensitive Drosophila melanogaster nicotinic acetylcholine receptor identified through chemically induced target site resistance and resistance gene identification. Insect Biochem. Molec. Biol. 40, 376-384 (2010).

21. Sheehan LS, Lill RE, Wilkinson B, Sheridan RM, Vousden WA, Kaja AL, Crouse GD, Gifford J, Graupner P, Karr L, Lewer P, Sparks TC, Leadlay PF, Waldron C, Martin CJ, Engineering the spinosyn PKS: directing starter unit incorporation. J Nat Prod 69:17021710 (2006).

22. Daeuble J, Sparks TC, Johnson P, Graupner PR, Modification of the butenyl-spinosyns utilizing cross-metathesis. Bioorg. Med. Chem. 17, 4197-4205 (2009).

23. Crouse GD, Sparks TC, McLeod CL, Demeter DA, Bryan K, Brown AV, Dent WH III, Cudworth DP, Nugent JS, Hunter R, Samaritoni JG, Pesticidal heterocyclic aromatic compounds prepared with pyranose intermediates PCT Int. Appl., WO2009102736 A1, US 9006468. 228 pages (2009).

24. Crouse GD, Sparks TC, McLeod CL, Demeter DA, Benko ZL, Camper DL, Pesticidal compositions of pyrimidine derivatives, U.S. Pat. Appl. Publ., US 20100204164 A1 20100812. 25 pages (2010). 


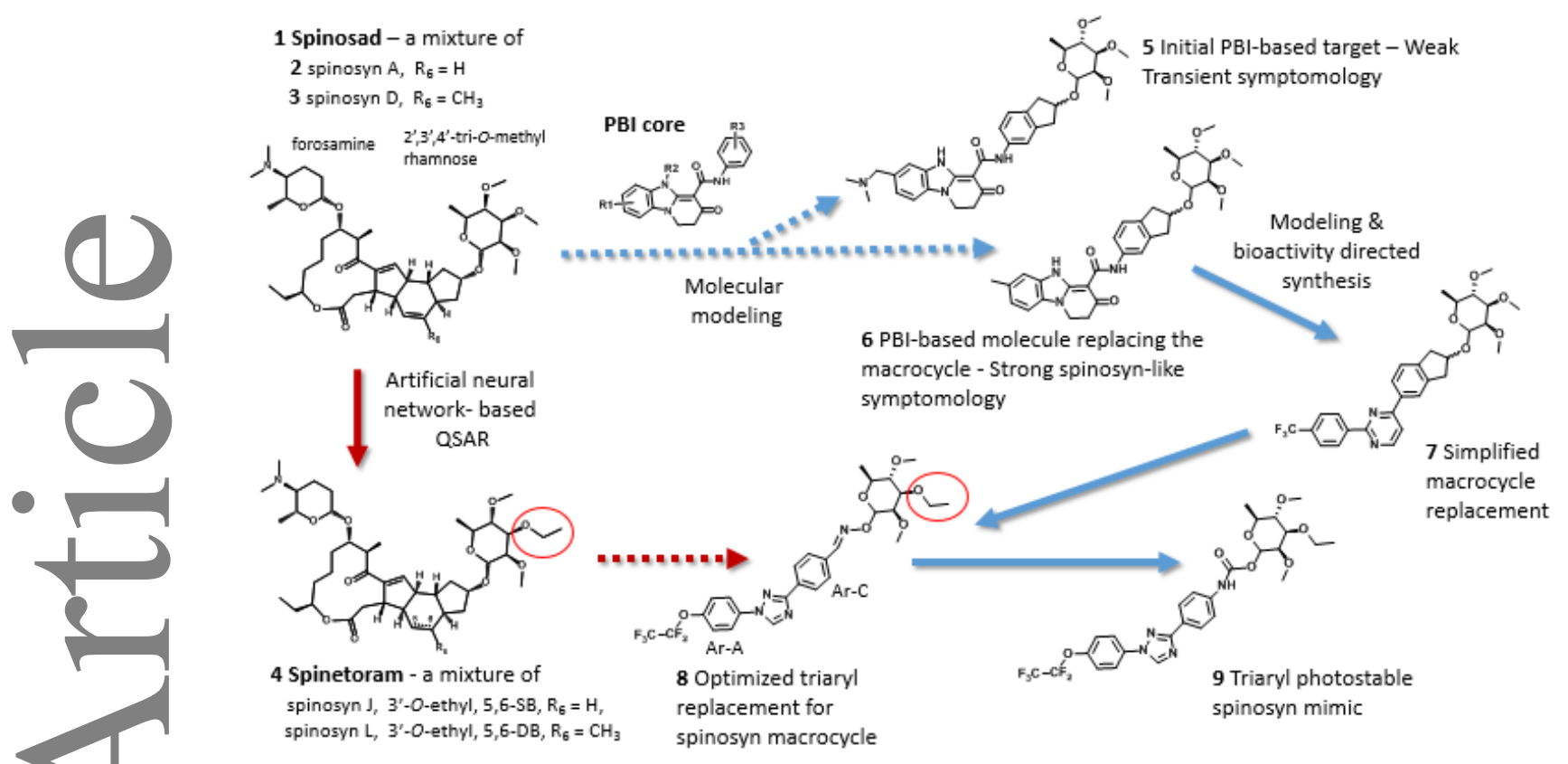

Fig. 1. Structures of commercial insecticides, spinosad 1 and spinetoram 4, and a conceptual route to the discovery of the synthetic spinosyn mimics. Spinosyn mimics were synthesized as described by Crouse et al. ${ }^{13,23,24}$ 

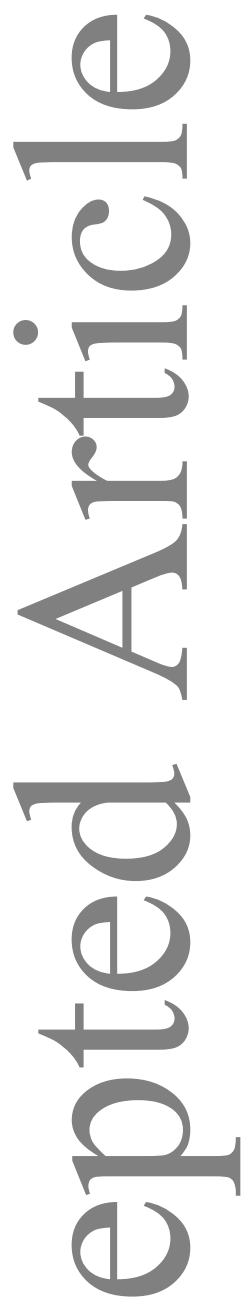

.
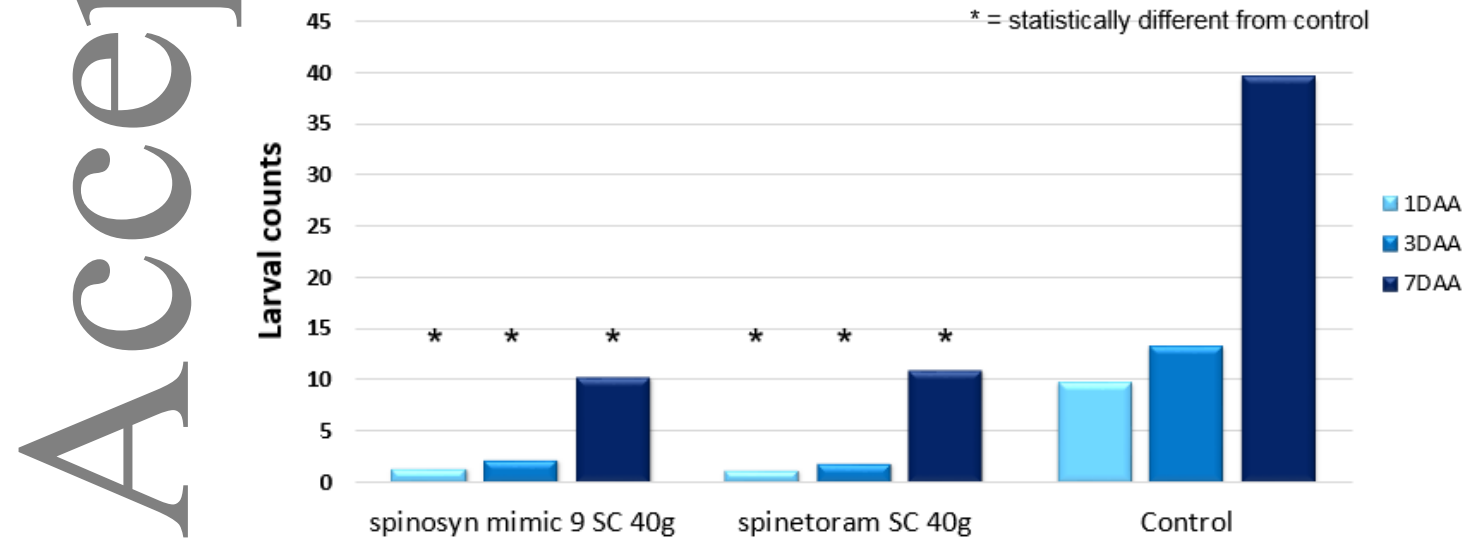

Fig. 3. Field efficacy of the synthetic spinosyn mimic 9 compared to spinetoram against larvae of Trichoplusia ni (cabbage looper) on cabbage in Wayside MS. *Statistically different from control, LSD $(\mathrm{P}=0.05)$.

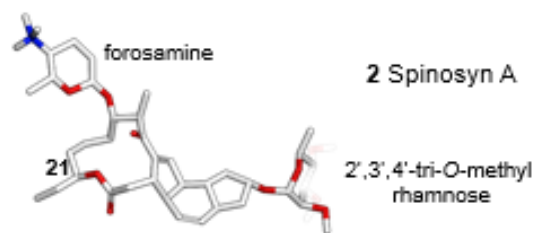

5 PBI core (orange) initial target

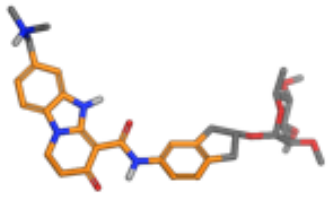

Overlay 1

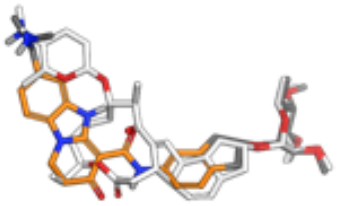

5 PBI core initial

target on 2 spinosyn A

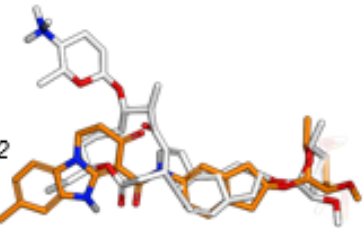

PBl-based macrocycle

Fig. 2. 3D-structures and overlay of spinosyn A 2, the PBI core 5, the initial PBI-based spinosyn mimic 6 and the optimized synthetic spinosyn mimic 9 possessing a triaryl replacement for the spinosyn macrocycle. Structures 6 \& 9 are aligned to the 2',3',4'-tri-O-methyl rhamnose of spinosyn A 2.

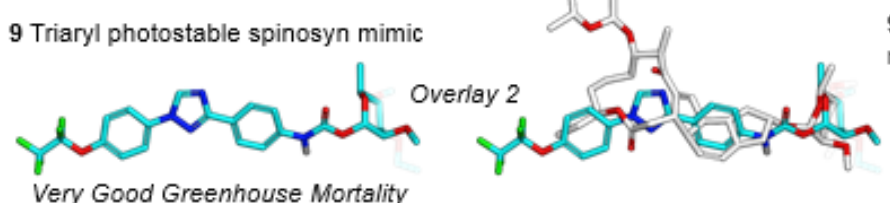

9 Triaryl photostable spinosyn mimic on 2 spinosyn A
6 PBI-based macrocycle replacement replacement on $\mathbf{2}$ spinosyn $\mathrm{A}$ 
Table 1. Insecticidal activity of spinosad, spinetoram, and selected synthetic spinosyn mimics to larvae of $S$. exigua $(\mathrm{Se}), \mathrm{H}$. zea $(\mathrm{Hz})$, and adults of two strains (WT and spinosad-resistant) of D. melanogaster $(D m)$.

\begin{tabular}{|c|c|c|c|c|c|c|}
\hline Assay $^{a}$ & $1^{b}$ & $4^{b}$ & 6 & 7 & 8 & 9 \\
\hline $\begin{array}{l}\text { Se. } \mathrm{LC}_{50} \mu \mathrm{g} / \mathrm{cm}^{2} \\
(95 \% \mathrm{FL})^{3}\end{array}$ & $\begin{array}{l}0.052 \\
(0.021- \\
0.083)\end{array}$ & $\begin{array}{l}0.0077 \\
(0.0044- \\
0.015)\end{array}$ & $>12.5$ & $<12.5^{\mathrm{g}}$ & $\begin{array}{l}0.0046 \\
(0.0038- \\
0.0055)\end{array}$ & $\begin{array}{l}0.0052 \\
(0.0017- \\
0.0105)\end{array}$ \\
\hline $\begin{array}{l}\mathrm{Hz} . \mathrm{LC}_{50} \mu \mathrm{g} / \mathrm{cm}^{2} \\
(95 \% \mathrm{FL})^{\mathrm{c}}\end{array}$ & $\begin{array}{l}0.058 \\
(0.045- \\
0.075)\end{array}$ & $\begin{array}{l}0.0087 \\
(0.0073- \\
0.010)\end{array}$ & $>12.5$ & $<12.5^{\mathrm{g}}$ & $\begin{array}{l}0.0034 \\
(0.0029- \\
0.0040)\end{array}$ & $\begin{array}{l}0.0043 \\
(0.0036- \\
0.0052)\end{array}$ \\
\hline $\begin{array}{l}\text { WT Dm }{ }^{\mathrm{d}} \mathrm{LC}_{50} \\
\text { ppm } \\
(95 \% \mathrm{FL})\end{array}$ & $\begin{array}{l}0.035 \\
(0.009- \\
0.123)\end{array}$ & $\begin{array}{l}0.025 \\
(0.0204- \\
0.032)\end{array}$ & -- & -- & $\begin{array}{l}0.0048 \\
(0.0035- \\
0.0057)\end{array}$ & $\begin{array}{l}0.0306 \\
(0.0252- \\
0.0373)\end{array}$ \\
\hline $\begin{array}{l}S R \mathrm{Dm}^{\mathrm{e}} \mathrm{LC}_{50} \\
\text { ppm } \\
(95 \% \mathrm{FL})\end{array}$ & $\begin{array}{l}10.9 \\
(9.29-13.4)\end{array}$ & $\begin{array}{l}3.52 \\
(2.79-4.38)\end{array}$ & -- & -- & $\begin{array}{l}0.55 \\
(0.39-0.72)\end{array}$ & $\begin{array}{l}3.75 \\
(3.07-4.46)\end{array}$ \\
\hline$R R^{f}$ & 311 & 139 & -- & -- & 114 & 123 \\
\hline $\begin{array}{l}\text { Greenhouse UV } \\
\text { Se LC }{ }_{50} \text { gai/ha } \\
\text { Day } 2^{\text {h }} \\
\text { Day } 4 \\
\text { Day } 7\end{array}$ & $\begin{array}{l}-- \\
-- \\
--\end{array}$ & $\begin{array}{l}3.0 \\
1.5 \\
3.5\end{array}$ & $\begin{array}{l}-- \\
-- \\
--\end{array}$ & $\begin{array}{l}-- \\
-- \\
--\end{array}$ & $\begin{aligned} & 1.3 \\
> & 10 \\
> & 10\end{aligned}$ & $\begin{array}{l}0.9 \\
1.3 \\
1.8\end{array}$ \\
\hline
\end{tabular}

a Data for compounds $\mathbf{1}, \mathbf{4}, \mathbf{6 , 8}$ against $S e, H z$, WT Dm, SR Dm adapted from ${ }^{13}$

${ }^{\mathrm{b}} \mathbf{1}$ = spinosad, $\mathbf{4}$ = spinetoram

${ }^{c} \mathrm{FL}=$ fiducial limits

${ }^{d}$ Wild type (susceptible) adult D. melanogaster.

${ }^{\text {e }}$ Spinosad-resistant adult $D$. melanogaster - see reference 9 for details.

${ }^{f}$ Resistance ratio: $\mathrm{LC}_{50}$ SR-Dm / LC 50 WT Dm.

g $100 \%$ mortality - lowest dosage tested

${ }^{\mathrm{h}}$ Days post treatment - plants were treated and then held under UV conditions - larvae were placed on treated leaves at internals of 2, 4 and 7 days post-treatment and then held 5 days after which mortality was determined. 